\title{
ALGEBRAIC CHARACTERISTIC CLASSES FOR IDEMPOTENT MATRICES
}

\author{
FRANCISCO GÓMEZ
}

To the memory of Pere Menal

\begin{abstract}
This paper contains the algebraic analog for idempotent matrices of the Chern-Weil theory of characteristic classes. This is used to show, algebraically, that the canonical line bundle on the complex projective space is not stably trivial. Also a theorem is proved saying that for any smooth manifold there is a canonical epimorphism from the even dimensional algebraic de Rham cohomology of its algebra of smooth functions onto the standard even dimensional de Rham cohomology of the manifold.
\end{abstract}

\section{Introduction}

In this paper I comment about algebraic characteristic classes and some related problems.

The idea for defining characteristic classes for finitely gencrated projective modules comes from the equivalence between vector bundles and finitely generated projective modules given by the cross section functor, see Swan [12]. These classes were defined by Ozeki, [9], by imitating the Chern Weil construction via principal connections. The classes introduced by Ozeki belonged to a cohomology of the ring based on the derivations of such a ring. Later Kong, [7], defined the Euler class for inner product projective modules by using linear connections on the module and the algebraic de Rham cohomology of the ring, i.e. the cohomology of the exterior algebra of the Kähler differentials with the canonical extension of the universal derivative. See also Karoubi, [6], for a generalization to the noncommutative case and its relation with the corresponding theory using cyclie homology, see Connes, [2].

The functors $A \rightarrow P_{A}, A \rightarrow I_{A}$ are naturally equivalent, where $A \rightarrow P_{A}$ associates to each commutative ring with unit, $A$, the isomorphism class of finitely generated projective modules over $A$, and $A \rightarrow I_{A}$ 
associates to such a ring $A$ the equivalence class of idempotent matrices with entries in $A$, where an idempotent matrix $\varphi$ is equivalent to an idempotent matrix $\varphi^{\prime}$ if and only if there exist matrices $M$ and $N$ such that $M N=\varphi, N M=\varphi^{\prime}$. This equivalence is obtained by sending the class of $\varphi$ in $I_{A}$ to the class of the image of $\varphi$ in $P_{A}$. The above equivalence induces one between the abelian group $\tilde{K}_{0}(A)$ of stably isomorphism classes of finitely generated projective modules and the corresponding abelian group obtained from the idempotent matrices. The opposite of the class represented by an idempotent matrix $\varphi$ is clearly the class represented by $I_{n}-\varphi$, where $I_{n}$ is the $n \times n$ identity matrix.

2. Examples.

a) Consider the idempotent matrix

$$
\varphi=\left(\begin{array}{ccc}
1-X^{2} & -X Y & -X Z \\
-X Y & 1-Y^{2} & -Y Z \\
-X Z & -Y Z & 1-Z^{2}
\end{array}\right)
$$

with entries in $A=R[X, Y, Z] /\left(X^{2}+Y^{2}+Z^{2}-1\right)$, where $R$ is any commutative ring with unit.

The class $\varphi$ in $\tilde{K}_{0}(A)$ is zero. In fact the opposite class is represented by the idempotent matrix

$$
\left(\begin{array}{ccc}
X^{2} & X Y & X Z \\
X Y & Y^{2} & Y Z \\
X Z & Y Z & Z^{2}
\end{array}\right)=M N
$$

with $M=\left(\begin{array}{c}X \\ Y \\ Z\end{array}\right), N=\left(\begin{array}{lll}X & Y & Z\end{array}\right)$ and we have $N M=(1)$.

If $A$ is the ring of $C^{\infty}$ functions on the 2-sphere $S^{2}$, the above matrix $\varphi$ represents the tangent bundle of $S^{2}$ and this example is the algebraic version of the well kown fact that the tangent bundle of the 2-sphere is stably trivial.

b) If $R=\mathbf{R}$ (real numbers) or $R=\mathbf{C}$ (complex numbers), the Grassmannian of r-planes in $R^{n}, G_{r}\left(R^{n}\right)$, is diffeomorphic to the manifold whose points are the $n \times n$ idempotent matrices $\varphi=\left(\varphi_{i}^{j}\right)$ such that $\bar{\varphi}=\varphi^{t}$ and having rank $r$ ( $\bar{\varphi}$ denotes conjugate and $\varphi^{t}$ transpose). The canonical r-plane bundle $\gamma$ is then represented by the $n \times n$ idempotent matrix $\gamma=\left(\gamma_{i}^{j}\right)$ where $\gamma_{i}^{j}: G_{r}\left(R^{n}\right) \rightarrow R$ is given by $\gamma_{i}^{j}(\varphi)=\varphi_{i}^{j}$. Therefore if $R=\mathbf{R}$ (resp. $R=\mathbf{C}$ ) the idempotent matrix $\gamma$ makes sense in the quotient of the polynomial ring $R\left[X_{i}^{j}\right]_{i, j=1, \ldots, n}$ (resp. $R\left[i\left[\mid X_{i}^{j}, Y_{i}^{j}\right]_{i, j=1, \ldots, n}\right.$ ) by the ideal gencrated by the polinomial identities for the conditions 
$\gamma^{2}=\gamma, \bar{\gamma}=\gamma^{t}$ and $\operatorname{rank} \gamma=r$. This allows us to define what a canonical $r$-plane bundle should be in general for any commutative ring with unit $R$ ( resp. $R[i]$ with $i^{2}=-1$ ).

For instance if $r=1, n=2$ the ring is the quotient of $R[i]\left[X_{i}^{j}, Y_{i}^{j}\right]_{i, j=1,2}$ by the ideal generated by $Y_{1}^{1}, Y_{2}^{2}, X_{1}^{2}-X_{2}^{1}, Y_{1}^{2}+Y_{2}^{1}, X_{1}^{1}+X_{2}^{2}-1$, $X_{1}^{1}\left(1-X_{1}^{1}\right)-\left(\left(X_{1}^{2}\right)^{2}+\left(Y_{1}^{2}\right)^{2}\right)$; which is isomorphic to

$$
R[i]\left[X_{1}^{1}, X_{1}^{2}, Y_{1}^{2}\right] /\left(X_{1}^{1}\left(1-X_{1}^{1}\right)-\left(\left(X_{1}^{2}\right)^{2}+\left(Y_{1}^{2}\right)^{2}\right)\right.
$$

and the algebraic version of the canonical complex linc bundle should be the idempotent matrix

$$
\gamma=\left(\begin{array}{cc}
X_{1}^{1} & X_{1}^{2}+i Y_{1}^{2} \\
X_{1}^{2}-i Y_{1}^{2} & 1-X_{1}^{1}
\end{array}\right)
$$

If $\frac{1}{2} \in R$ we can write $X=2 X_{1}^{2}, Y=2 Y_{1}^{2}, Z=2 X_{1}^{1}-1$ and the ring above is isomorphic to $A=R[i][X, Y, Z] /\left(X^{2}+Y^{2}+Z^{2}-1\right)$, where $R$ is any integral domain containing $\frac{1}{2}$.

Choose now any commutative differential graded algebra $(\Omega, d)$ with $\Omega^{0}=A$. For instance the algcbraic de Rham complex, i.e. $\Omega^{0}=A, \Omega^{1}$ are the Kähler differentials with the universal derivative $d: \Omega^{0} \rightarrow \Omega^{1}$, $\Omega^{p}$ is the p-th exterior power of $\Omega^{1}$ and $d: \Omega^{p} \rightarrow \Omega^{p+1}$ is the canonical extension of $d: A \rightarrow \Omega^{1}$. In our case is clear that $\Omega^{p}=0$ for $p \geq 3$ and $\Omega^{2}=A . \omega$ is a free module of rank one, where $\omega=X d Y d Z+Y d Z d X+$ $Z d X d Y$.

It makes sense now to consider the matrix $\gamma(d \gamma)^{2}$ with cntries in $\Omega^{2}$ and a straightforward computation yields trace $\left(\gamma(d \gamma)^{2}\right)=-\frac{i}{2} \omega$.

Observe that for a matrix $\alpha$ with entries in $R[i]$ one has $d \alpha=0$ and for a matrix $\psi=\left(\begin{array}{cc}\gamma & 0 \\ 0 & I_{m}\end{array}\right)$ where $I_{m}$ is the $m \times m$ identity matrix, one has trace $\psi(d \psi)^{2}=\operatorname{trace}\left(\gamma(d \gamma)^{2}\right)$. These two observations and the following lemma tell us that to prove that $\gamma$ does not represent the zero class in $\bar{K}_{0}(A)$ we must show that trace $\left(\gamma(d \gamma)^{2}\right)$ is not of the form $d \alpha$ for some $\alpha \in \Omega^{1}$. In our case we must show that $\omega \notin \operatorname{Im}(d)$.

Lemma. If $\varphi$ and $\varphi^{\prime}$ are idempotent matrices with $M N=\varphi, N M=$ $\varphi^{\prime}$ for some matrices $M, N$; then $\operatorname{trace}\left(\varphi^{\prime}\left(d \varphi^{\prime}\right)^{2}\right)-\operatorname{trace}\left(\varphi(d \varphi)^{2}\right)=$ $d(\operatorname{trace}(\varphi M d N))$.

Proof:

$$
\begin{aligned}
& \operatorname{trace}\left(\varphi^{\prime}\left(d \varphi^{\prime}\right)^{2}\right)-\operatorname{trace}\left(\varphi(d \varphi)^{2}\right)= \\
= & \operatorname{trace}\left(N M(d N \cdot M+N \cdot d M)^{2}-M N(d M \cdot N+M \cdot d N)^{2}\right)= \\
= & \operatorname{trace}\left(\varphi d M d N-\varphi^{\prime} d N d M-2 \varphi d M \varphi^{\prime} d N\right) .
\end{aligned}
$$


The last equality comes from the observation that for $\alpha, \beta$ matrices with entries in $\Omega^{p}, \Omega^{q}$ respectively one has trace $(\alpha \beta)=(-1)^{p q} \operatorname{trace}(\beta \alpha)$. In particular trace $\left(\alpha^{2}\right)=0$ for $p$ odd.

On the other hand

$$
\begin{aligned}
& d(\operatorname{trace}(\varphi M d N))=\operatorname{trace}(d(M N M d N))= \\
& =\operatorname{trace}(d M \cdot N M d N+M d N \cdot M d N+M N d M \cdot d N)= \\
& =\operatorname{trace} \varphi d M d N-\operatorname{trace} \varphi^{\prime} d N d M .
\end{aligned}
$$

Therefore to conclude the proof of the lemma one must show that $\operatorname{trace}\left(\varphi d M \varphi^{\prime} d N=0\right.$.

But we have

$$
\begin{aligned}
& \operatorname{trace}\left(\varphi d M \varphi^{\prime} d N\right)=\operatorname{trace}\left(\varphi^{2} d M \varphi^{\prime} d N\right)= \\
= & \operatorname{trace}\left(M \varphi^{\prime} N d M \varphi^{\prime} d N\right)=\operatorname{trace}\left(\varphi^{\prime} N d M \varphi^{\prime} d N . M\right)= \\
= & \operatorname{trace}\left(\varphi^{\prime} N d M \varphi^{\prime} d \varphi^{\prime}\right)-\operatorname{trace}\left(\varphi^{\prime} N d M \varphi^{\prime} N d M\right)= \\
= & \operatorname{trace}\left(\varphi^{\prime} N d M \varphi^{\prime} d \varphi^{\prime}\right)=\operatorname{trace}\left(N d M \varphi^{\prime} d \varphi^{\prime} \cdot \varphi^{\prime}\right)=0,
\end{aligned}
$$

because $\varphi^{\prime} d \varphi^{\prime} \cdot \varphi^{\prime}=0$.

In fact, $d \varphi^{\prime}=d\left(\varphi^{\prime}\right)^{2}=d \varphi^{\prime} \cdot \varphi^{\prime}+\varphi^{\prime} d \varphi^{\prime}$ implies $d \varphi^{\prime} \cdot \varphi^{\prime}=d \varphi^{\prime} \cdot \varphi^{\prime}+$ $\varphi^{\prime} d \varphi^{\prime} \cdot \varphi^{\prime}$

Hence $\varphi^{\prime} d \varphi^{\prime} . \varphi^{\prime}=0$

If we assume that $R$ contains the rational numbers, then we may show that $\omega \notin \operatorname{Im}(d)$ by considering the $R[i]$-linear map given by Kong, cf. page 297 of [7], $\rho: \Omega^{2} \rightarrow R[i]$ given by $\rho(\omega)=1, \rho\left(X^{\alpha} Y^{\beta} \cdot \omega\right)=0$ if $\alpha$ or $\beta$ are odd, $\rho\left(X^{\alpha} Y^{\beta} Z \cdot \omega\right)=0, \rho\left(X^{2 n} Y^{2 m} \cdot \omega\right)=\frac{1}{2(n+m)+1} \cdot \frac{(2 m) !(2 n) !}{2(m+n) !} \cdot \frac{(m+n) !}{m ! n !}$ and check that $\rho$ vanishes on the image of $d$.

Actually it is not difficult to give a direct proof showing that $\omega$ is not in the image of $d$ for any ring $R[i]$ as we have considered, i.e. an integral domain containing $\frac{1}{2}$.

\section{Characteristic classes for idempotent matrices}

The examples above suggest how to define characteristic classes for idempotent matrices $\varphi$ having entries in an $R$-algebra $A$ where both $R$ and $A$ are commutative rings with the same unit element. One simply chooses a commutative graded differential algebra $(\Omega, d)$ with $\Omega^{0}=A$. For instance take $(\Omega, d)$ as the algebraic de Rham complex of $A$. Then $\operatorname{det}\left(\varphi(d \varphi)^{2}+I_{n}\right)=1+c_{1}(\varphi)+\cdots+c_{n}(\varphi)$, where $I_{n}$ is the identity $n \times n$ matrix and $c_{p}(\varphi) \in \Omega^{2 p}$ is the p-th characteristic coefficient of $\varphi$.

Define also $\operatorname{Tr}_{p}(\varphi)=\operatorname{trace}\left(\varphi(d \varphi)^{2}\right)^{p} \in \Omega^{2 p}$ for $p \geq 1$ (the trace coefficient of $\varphi$ ). They are related by $\operatorname{Tr}_{p}=Q_{p}\left(C_{1}, \ldots, C_{p}\right), p ! C_{p}=$ $P_{p}\left(\operatorname{Tr}_{1}, \ldots, \operatorname{Tr}_{p}\right)$ for polynomials $P_{i}, Q_{i}$ in $\mathrm{Z}\left[X_{1}, \ldots, X_{i}\right]$. 
Lemma. $d \operatorname{Tr}_{p}(\varphi)=0$. Thus if $R$ contains the rationals one has $d c_{p}(\varphi)=0$.

Proof: We have already seen that $\varphi d \varphi \cdot \varphi=0$ and $\varphi(d \varphi)^{2}=(d \varphi)^{2} \varphi$.

Observe that we have $\operatorname{trace}\left(\varphi(d \varphi)^{2 p+1}\right)=0$.

In fact,

$$
\varphi(d \varphi)^{2 p+1}=\varphi^{2}(d \varphi)^{2 p+1}
$$

Thus

$\operatorname{trace}\left(\varphi(d \varphi)^{2 p+1}\right)=\operatorname{trace}\left(\varphi(d \varphi)^{2 p+1} \varphi\right)=\operatorname{trace}\left(\varphi d \varphi \cdot \varphi \cdot(d \varphi)^{2 p}\right)=0$.

But

$$
(d \varphi)^{2 p+1}=d \varphi \cdot \varphi \cdot(d \varphi)^{2 p}+\varphi(d \varphi)^{2 p+1}
$$

Therefore

$$
\operatorname{trace}(d \varphi)^{2 p+1}=\operatorname{trace}\left(d \varphi \cdot \varphi \cdot(d \varphi)^{2 p}\right)=\operatorname{trace}\left(\varphi(d \varphi)^{2 p+1}\right)=0
$$

As a consequence of this lemma $c_{p}(\varphi)$ represents a cohomology class in $H^{2 p}(\Omega, d)$ called the pth-Chern class of $\varphi$. In the example above we have computed the first Chern class of $\gamma$.

If $R=\mathbf{R}, A=C^{\infty}(X)$ and $\varphi$ is the idempotent matrix corresponding to a vector bundle $\xi$ over the $C^{\infty}$ manifold $X$, one has that the cohomology class represented by $c_{p}(\varphi)$ is zero for $p$ odd and the cohomology class represented by $c_{2 q}(\varphi)$ equals $(2 \pi)^{2 q} . p_{q}(\xi)$, where $p_{q}(\xi)$ is the q-th Pontrjagin class of $\xi$. If $R=\mathbf{C}, A=C^{\infty}(X ; \mathrm{C})$ and $\varphi$ corresponds to a complex vector bundle $\xi$, the cohomology class represented by $c_{p}(\varphi)$ equals $(-2 \pi i)^{p} c_{p}(\xi)$, where $c_{p}(\xi)$ is the $\mathrm{p}$-th Chern class of $\xi$.

The next proposition shows that actually the p-th Chem class is defined for elements in $\tilde{K}_{0}(A)$.

Proposition. If $\varphi$ and $\varphi^{\prime}$ are equivalent idempotent matrices, then $c_{p}\left(\varphi^{\prime}\right)-c_{p}(\varphi)$ belongs to the image of $d$.

Proof: Suppose $M N=\varphi, N M=\varphi^{\prime}$. We proceed as follows. Observe first that the images of $\varphi$ and $\varphi^{\prime}$ are finitely generated projective modules and that $N: \operatorname{Im}(\varphi) \rightarrow \operatorname{Im}\left(\varphi^{\prime}\right)$ is an isomorphism with inverse $M$. Define 
then linear connections $\nabla: \operatorname{Im}(\varphi) \rightarrow \operatorname{Im}(\varphi) \otimes \Omega^{1}, \nabla^{\prime}: \operatorname{Im}\left(\varphi^{\prime}\right) \rightarrow$ $\operatorname{Im}\left(\varphi^{\prime}\right) \otimes \Omega^{1}$ and $\tilde{\nabla}: \operatorname{Im}\left(\varphi^{\prime}\right) \rightarrow \operatorname{Im}\left(\varphi^{\prime}\right) \otimes \Omega^{1}$ as follows:

$$
\nabla x=\sum_{i=1}^{n} d x_{i} \otimes \varphi\left(e_{i}\right)
$$

for $x=\sum_{i=1}^{n} x_{i} e_{i} \in \operatorname{Im}(\varphi) \subset A^{n}$, where $e_{1}, \ldots, e_{n}$ is the canonical basis of $A^{n}$.

$$
\nabla^{\prime} x^{\prime}=\sum_{i=1}^{m} d x_{i}^{\prime} \otimes \varphi^{\prime}\left(e_{i}^{\prime}\right)
$$

for $x^{\prime}=\sum_{i=1}^{m} x_{i}^{\prime}, e_{i}^{\prime} \in \operatorname{Im}(\varphi) \subset A^{m}$, where $e_{1}^{\prime}, \ldots, e_{n}^{\prime}$ is the canonical basis of $A^{m}$.

$\tilde{\nabla}$ such that the following diagram commutes

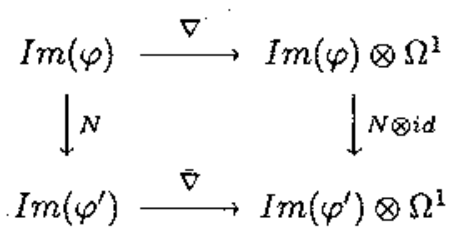
$\tilde{\nabla}$.

Denote by $R_{\nabla}, R_{\nabla^{\prime}}$ and $R_{\bar{\nabla}}$ the corresponding curvatures for $\nabla, \nabla^{\prime}$,

Remark. Recall that if $\nabla: M \rightarrow \Omega^{1} \otimes M$ is a lincar connection for a finitely generated projective A-module $M$, then $\nabla^{2}: M \rightarrow \Omega^{2} \otimes M$ is A-linear and so it can be regarded as an element $R_{\nabla}$ of $\Omega^{2} \otimes L_{M}$ which is called the curvature of $\nabla$. If $\nabla$ denotes also the induced linear connection on $L_{M}$ and the corresponding covariant exterior derivative (just imitating the usual defnitions in Differential Geometry) one has the Bianchi identity $\nabla R_{\nabla}=0$.

An easy computation shows that trace $\left(R_{\nabla} \circ \cdots \circ R_{\nabla}\right)=\operatorname{trace}\left(\varphi(d \varphi)^{2 p}\right)$, $\operatorname{trace}\left(R_{\nabla^{\prime}} \circ \cdots \circ R_{\nabla^{\prime}}\right)=\operatorname{trace}\left(\varphi^{\prime}\left(d \varphi^{\prime}\right)^{2 p}\right)$ and $\operatorname{trace}\left(R_{\nabla} \circ \cdots \circ R_{\nabla}\right)=$ $\operatorname{trace}\left(R_{\tilde{\nabla}} \circ \cdots \circ R_{\tilde{\nabla}}\right)$.

Therefore to finish the proof we must check that for any two linear connections $\nabla_{1}, \nabla_{2}$ on a finitely generated projective module, we have that trace $\left(R_{\nabla_{1}} \circ \cdots \circ R_{\nabla_{1}}\right)-\operatorname{trace}\left(\left(R_{\nabla_{2}} \circ \cdots \circ R_{\nabla_{2}}\right)\right.$ belongs to the image of $d$. But since $\nabla_{2}-\nabla_{1} \in \Omega^{1} \otimes L_{M}$, it is enough to do it for $\nabla_{2}=$ $\nabla_{1}+\alpha \otimes \psi$ with $\psi \in L_{M}, \alpha \in \Omega^{1}$. In this case $R_{\nabla_{i}}=R_{\nabla_{1}}+\nabla(\alpha \otimes \psi)$ and the proof is an easy consequence of Bianchi identity and the relation $\nabla \circ$ trace $=$ trace $\circ d$. 


\section{Some questions}

a) Suppose $A=C^{\infty}(X)$, where $X$ is a $C^{\infty}$-manifold, we have then a canonical homomorphism $H_{d R}^{*}\left(C^{\infty}(X)\right) \rightarrow H_{d R}^{*}(X)$ where $H_{d R}^{*}\left(C^{\infty}(X)\right)$ denotes the algebraic de Rham cohomology of $C^{\infty}(X)$ and $H_{d R}^{*}(X)$ denotes the usual de Rham cohomology of $X$.

It is well known that the above homomorphism is not, in general, an isomorphism, see proposition 6 , page 143 of [10], but we have the following theorem

Theorem. The homomorphism $H_{d R}^{*}\left(C^{\infty}(X)\right) \rightarrow H_{d R}^{*}(X)$ is an epimorphism in even dimensions.

Proof: Observe that if $\xi$ is a vector bundle over $X$ the algebraic Chern classes of the corresponding idempotent matrix are mapped canonically to the corresponding characteristic classes of $\xi$. Then we use the fact that any even dimensional de Rham class, with coefficients in $\mathbf{Q}, \mathbf{R}$ or $\mathrm{C}$ is the characteristic class of some complex vector bundle over $X$. This is true because of the isomorphism given by the Chern character $c h: K(X) \otimes \mathbf{z} \mathbf{Q} \rightarrow \prod_{p \geq 0} H^{2 p}(X ; \mathbf{Q})$, see page 119 of $[\mathbf{3}]$.

We can pose then the question: what happens in odd dimensions?

b) It seems natural to consider for a topological space $X$ the algebraic de Rham cohomology of its algebra of real or continuous functions. What can be said of such a cohomology? Except for $H^{0}$ or trivial cases, see [4], I do not know anything else.

c) It should be interesting to find, for a given space, commutative differential graded algebra $(\Omega, d)$ such that $\Omega^{0}=A$ is some subalgebra of functions of $X$ such that we have isomorphisms $H_{d R}(\Omega, d) \cong H^{*}(X)$ and $\bar{K}_{0}(A) \cong \tilde{K}_{0}(X)$. See also Carral [1],Lønsted [8] and Swan [11] for some other related problems.

\section{References}

1. M. Carral, Modules projectives sur les anneaux des fonctions, J. of Algebra 87 (1984), 202-212.

2. A. Connes, Noncommutative differential geometry, Publ. Math. I.H.E.S. $62(1985), 257-360$.

3. E. Dyer, "Cohomology theories," Benjamin, 1969.

4. F. Gómez, The number of generators of the algebra of Kähler differentials, Demonstratio Math. 23 (1990), 375-383. 
5. F. GómEz, Diferenciales de álgebras de funciones continuas y clases características de módulos proyectivos finitamente generados, Colloquium 86-88, Matemáticas, Univ. Extremadura (1988), 52-58.

6. M. Karovbi, Homologic cyclique et $K$-theorie, Asterisque 149 (1987).

7. M. KONG, Euler classes of Inner Product Modules, J. of Algebra 49 (1977), 276-303.

8. K. LøNSTED, Vector bundles over finite $C W$ complexes are algebraic, Proc. A.M.S. 38, 1 (1973), pp 27-31.

9. H. OzEKI, Chern classes of projective modules, Nagoya Math. J. 23 (1963), $121-152$.

10. H. A. Osborn, Derivations of commutative algebras, Illinois $J .13$ (1969), 137-144.

11. R. SWAN, Topological examples of projective modules, Trans. A.M.S. 230 (1977), 201-234.

12. R. SWAN, Vector bundles and projective modules, Trans. A.M.S. $105(1962), 264-277$.

Departamento de Álgebra, Geometría y Topología

Facultad de Ciencias

Universidad de Málaga

Campus Teatinos, Apartado 59

29080 Málaga

SPAIN

Rebut el 7 de Gener de 1992 